\title{
Sistema de control de temperatura on-off implementado en el proceso de termoformado para elaboración de bloque a partir de envases multicapas
}

\section{On-off temperature control system implemented in the thermoforming process for block production from multi-layer containers}

ROSALES-DAVALOS, Jaime †*, ENRIQUEZ-PEREZ, Ma. de los Ángeles, LÓPEZ-RAMÍREZ, Roberto y MASTACHE-MASTACHE, Jorge Edmundo

Tecnológico de Estudios Superiores de Jocotitlán, Carretera Toluca-Atlacomulco KM 44.8, Ejido de San Juan y San Agustín Jocotitlán, 50700 Jocotitlán, Estado de México

ID $1^{\text {er }}$ Autor: Jaime, Rosales-Davalos / ORC ID: 0000-0002-9059-6093, CVU CONACYT ID: 812961

ID $1^{\text {er }}$ Coautor: Ma. de los Ángeles, Enriquez-Perez / ORC ID: 0000-0002-2280-0661, Researcher ID Thomson: H-93992018

ID $2^{\text {do }}$ Coautor: Roberto, López-Ramírez / ORC ID: 0000-0001-8341-3684, CVU CONACYT ID: 233228

ID $3^{\text {er }}$ Coautor: Jorge Edmundo, Mastache-Mastache / ORC ID: 0000-0001-6104-6764, Researcher ID Thomson: H-11872018, CVU CONACYT ID: 544943

DOI: $10.35429 / J E E .2019 .10 .3 .17 .21$

Recibido Junio 20, 2019; Aceptado Septiembre 30, 2019

\section{Resumen}

En este trabajo se diseñó e implementó un sistema de control de temperatura (SCT) en el proceso de termoformado para la fabricación de bloques para construcción de muros (BCM). El proceso de control en el SCT consiste en establecer la temperatura de referencia para el termoformado y enviarla al microcontrolador Mega 2560. Este componente procesa la señal de entrada, obteniendo seis señales digitales que controlan la temperatura de la superficie de cada una de las caras externas del molde del $B C M$ que posee una geometría de prisma rectangular. Estas señales son enviadas a la etapa de aislamiento eléctrico donde son excitados los actuadores habilitan seis resistencias eléctricas (200 Watts a 120 Volts). La retroalimentación de la temperatura en el sistema de control se realizó a través de termopares tipo$\mathrm{k}$, cuyas señales analógicas se comparan con la señal de referencia para regular la temperatura. Este proyecto contribuirá a establecer y controlar la temperatura de las resistencias, implementadas para el proceso de termoformado, para la elaboración de bloques utilizando envases multicapas.

Control, temperatura, termoformado

\begin{abstract}
In this work, a temperature control system (SCT) was designed and implemented in the thermoforming process for the manufacture of blocks for wall construction (BCM). The control process in the SCT consists in establishing the reference temperature for the thermoforming and send it to the Mega 2560 microcontroller. This component processes the input signal, obtaining six digital signals that control the surface temperature of each of the external faces of the BCM mold that has a rectangular prism geometry. These signals are sent to the electrical isolation stage where the actuators enable six electrical resistances (200 Watts to 120 Volts). The feedback of the temperature in the control system was made through type-k thermocouples, whose analog signals are compared with the reference signal to regulate the temperature. This project contributes to establish and control the temperature of the resistances, implemented for the thermoforming process, for the elaboration of blocks using multilayer containers.
\end{abstract}

Control, temperature, thermoforming

Citación: ROSALES-DAVALOS, Jaime, ENRIQUEZ-PEREZ, Ma. de los Ángeles, LÓPEZ-RAMÍREZ, Roberto y MASTACHE-MASTACHE, Jorge Edmundo. Sistema de control de temperatura on-off implementado en el proceso de termoformado para elaboración de bloque a partir de envases multicapas. Revista de Ingeniería Eléctrica. 2019. 3-10: 17-21

\footnotetext{
$\dagger$ Investigador contribuyendo como primer Autor.

*Correspondencia al Autor correo electrónico: (jaime.rosales@tesjo.edu.mx)
} 


\section{Introducción}

Inicialmente, la humanidad generaba residuos biodegradables con alto grado de descomposición, de manera que la naturaleza podía conservar su equilibrio. Poco a poco, con la nuevas tecnologías y maquinarias se fueron generando residuos no biodegradables que han amenazado la capacidad de autodepuración de la naturaleza, en consecuencia, creado la necesidad de disminuir el impacto negativo al medio ambiente (Bernard, 1999).

Por ello surgen nuevas alternativas para reusar los desechos generados de los envases multicapa. Es decir, la empresa tetra pak en México se ha propuesto que para el 2020, agregaran 24,000 envases de Tetra Pak para reciclar y se incrementara $35 \%$ al $40 \%$ su tasa de reciclaje en México (Tetra Pak busca aumentar su tasa de reciclaje, 2019).

Si se centra la atención en los residuos generados por estos envases, una de las formas de reciclaje de este producto es por el proceso de termformado donde el material es molido y aplicando una temperatura, la cual integra los componentes para formar un solo material. Un Un factor importante es el control de la temperatura en el termoformado, en la década de los 80's hasta la actualidad se ha implementado el proceso de termoformado en la elaboración de diversos productos (empaques, contenedores de comida, partes de automóviles, señalizaciones y anuncios, en la construcciónde los muros de las viviendas, equipaje y equipos fotográficos) apartir de pellet de resina; además de reciclar su desperdicio, donde todos los polímeros termoplásticos son adecuados.

El proceso consiste en el calentamiento de laminas de diferentes espesores que se les trasminte calor uniformemente. Este proceso puede ser suministrado por gas con circulación de aire y temperatura o por resistencias eléctricas entre $180^{\circ} \mathrm{C}$ a $200^{\circ} \mathrm{C}$ (PLASTIGLAS, 1990).

Los hornos están construidos de placas metálicas, en su interior hay resitencias eléctricas de una determinada potencia electrica para el calentamiento de los mismos. Las resitencias pueden ser de tipo "S" o "U", y sus caraterisiticas eléctricas varian de acuerdo a los requerimientos de la planta.
Y finalmente el control de temperatura de termoformado es importante para la producción y el cumplimiento con las normas de calidad INEN del producto (INEN, 2010). La calidad dimesional y defectos de acabados en productos son afectados a los amplios rangos de variación de temperatura en el proceso (Jiang \& Jiang, 2012).

Los sistemas de las plantas de termoformado la mayoría son no lineales. Esto se debe la forma de las resistencias que podrían producir una irradiación irregular de calor en el horno (Wang, 2012). Además, tambien interviene la posición del sensor de temperatura y el retardo del sistema de TUMBASEC. C. A.

Por los factores que interviene en el proceso de termoformado el sistema se convierte en un sistema complejo, por lo tanto, la mayoría de los sistema son ON-OFF, debido a que el método es mas sencillo de implementar (Jiang \& Jiang, 2012)

La maquinas de procesamiento de plástico en Ecuador utilizan controladores pirómetricos, las restantes implementan PLC's las cuales son programadas con controladores de tipo On-Off (Martínez \& Moya, 2015)

Como lo menciono Bernard, una alternativa para disminuir el impacto ambiental, es reutlizando los envases multicapa en la elaboración de aglomerados, por lo tanto, se diseño e implemento un sistema de control de temeperatura (SCT) On-Off el proceso de termoformado donde se fabrican bloques para la contrucción de muros (BCM) apartir de reciclar envases multicapa (contienen $75 \%$ de carton, $20 \%$ de polietileno y $5 \%$ de aluminio), los cuales son usados de manera indiscrimina y es inadecuada su disposición final; por lo tanto, se emplea el proceso de separación y reciclaje para la minimización del daño originado (Martínez Barrera, Hernández Zaragoza, López Lara, \& Menchaca Campos, 2015), los cuales pueden ser usados para la fabricación de muebles de oficina, ya que esta reportado que pueden reemplazar algunos artículos hechos de madera, disminuyendo la tala de madera (Inche Mitma, Vergiú Canto, Mavila Hinojoza, Godoy Martínez, \& Chung Pinzás, 2004). 
Las etapas del proceso para la fabricación de bloque son: a) Etapa de molienda que consiste generar hojuelas de tamaño de $3 \mathrm{~mm}$ aporximadamente apartir de las cajas o envases multicapas, esto se realiza con un sistema electromecánico de trituración, después se depositan las hojuelas de los envases multicapa en el interior del molde de geometría de un prisma rectangular, inmediatamente se le trasfiere temperatura al interior del molde a través de resistencias eléctricas; se establece el valor de referencia de temperatura de $170^{\circ} \mathrm{C}$ a $180^{\circ} \mathrm{C}$, donde el sistema de control manipula la temperatura, que se mentenga en el valor deseado, para la producción y el cumplimiento con las normas de calidad (INEN, 2010).

Este proceso se realiza en un periodo de 20 min y finalmente se procede a la etapa de enfriamiento del molde en conjunto con el bloque en un periodo de 20 a $30 \mathrm{~min}$. Finalmente, se extra el bloque resultante. Es por eso, que en este trabajo se reporta el diseño y construcción de un sistema de control cerrado On-Off para el control de tempertaura en el proceso de termoformado para la elaboración de bloques multicapas para su posible uso en la industria de la construcción.

\section{Descripción del método}

Para determinar la curva característica de la planta (resistencias eléctricas plana de 200 Watts a 120 Volts), la temperatura depende del tiempo; aplicando diferentes niveles de voltaje de corriente alterna en un periodo de $60 \mathrm{~min}$, partiendo la temperatura ambiental, después se determinaron los valores de las contantes de ganancia $(K)$, el tiempo de retardo $(L)$ y la constante de tiempo $(T)$ de cada una de las curva características de la resistencia eléctrica plana, implementando el método de Ziegle-Nichols, consistió en trazar una recta tangenta a la curva caracteristica de la resistencia electríca o implementado Matlab (Benjamin, 1996). para obtener la función de tranferencia de la planta, ver ecuación 1. Por consiguiente, se diseño e implemento el control On-Off utilizando el microcontrolador ATMEGA 2560 (software y base de datos), la etapa de actuadores esta constituida por optoacopladores, amplificadores y reles; finalmente se implemento el sistema de control On-Off basado en el diagrama de bloques, ver figura 1 , donde $U(t)$ es la señal de salida del controlador y $e(t)$ es la señal de error y $U_{1}$ es un valor máximo y $U_{2}$ es igual a cero.

$$
\frac{c(s)}{u(s)}=\frac{k e^{L s}}{T s+1}
$$

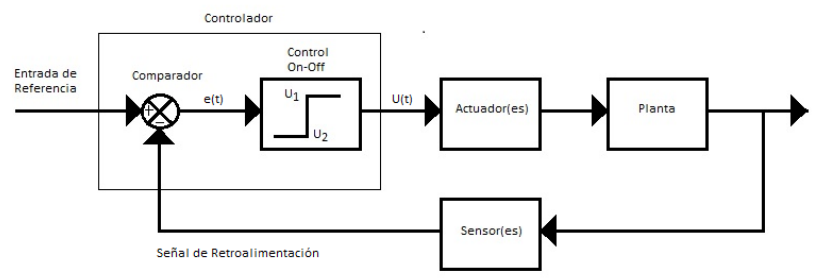

Figura 1 Diagrama de bloques de un controlador On-Off Fuente (Ogata, 1998)

El valor de la salida del controlador depende del error entre la señal de referencia y retroalimentación, si el error es mayor a cero la salida del controlador será el valor maximo, en caso contrario; es decir, el error es menor a cero la salida del controlador es un valor minimo, en realcion a la ecuación 2 y 3 (Ogata, 1998).

$$
\begin{aligned}
& U(t)=U_{1} \text { para } e(t)>0 \\
& U(t)=U_{2} \text { para } e(t)<0
\end{aligned}
$$

Se establecieron los rangos de entrada y salida de cada bloque de la figura 1. Finalmente, se obtuvieron las curvas del comportamiento del sistema a lazo cerrado On-Off, a diferente nivel valores referencia.

\section{Resultados}

En la figura 2, se muestran las curvas características de la planta para cuatro niveles de voltaje de corriente alterna 30, 60, 90 y $120 \mathrm{~V}_{\mathrm{AC}}$, al incrementar el voltaje de corriente alterna que se aplica a la resistencia eléctrica aumenta la temperatura, y disminuye el tiempo en el que se estabiliza la temperatura asi mismo la pendiente aumenta. En la tabla 1, se muestra el valor máximo de temperatura dependiendo de la tensión de corriente alterna suministrado a la planta.

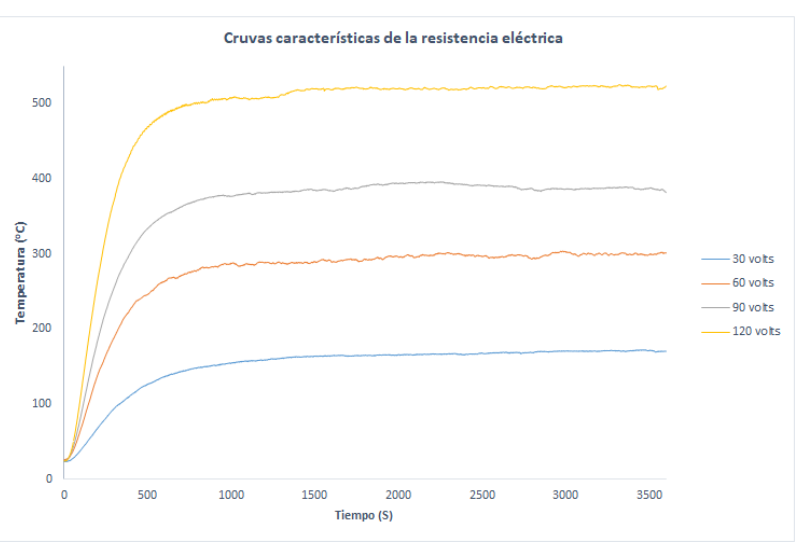

Figura 2 Curvas características de la resistencia eléctrica Fuente: Elaboración Propia 


\begin{tabular}{|r|r|}
\hline \multicolumn{2}{|c|}{ Rangos de Temperatura } \\
\hline Tensión $\left[\mathrm{V}_{\mathrm{AC}}\right]$ & $\begin{array}{c}\text { Temperatura } \\
{\left[{ }^{\circ} \mathrm{C}\right]}\end{array}$ \\
\hline 30 & 170 \\
\hline 60 & 300 \\
\hline 90 & 382 \\
\hline 120 & 523 \\
\hline
\end{tabular}

Tabla 1 Relación del voltaje con temperatura Fuente: Elaboración Propia

Posteriormente se obtienen los valores de la ganancia $(K)$, tiempo de retardo $(L)$ y constante de tiempo $(T)$, de las graficas de la figura 2 (tabla 2). Implemenetando el método Ziegle-Nichols.

\begin{tabular}{|r|c|r|c|}
\multicolumn{1}{c|}{} & \multicolumn{3}{c|}{ Valores de la constante } \\
\hline $\begin{array}{r}\text { Tensión } \\
\text { [VAC] }\end{array}$ & $K$ & \multicolumn{1}{c|}{$L$} & $T$ \\
\hline 30 & 5.5699 & -30 & 571.19 \\
\hline 60 & 4.9112 & -29.822 & 407.45 \\
\hline 90 & 4.2288 & -30 & 386.73 \\
\hline 120 & 4.0891 & -47.426 & 321.63 \\
\hline
\end{tabular}

Tabla 2 Constantes de la función de transferencia de la planta

Fuente: Elaboración Propia

Por lo tanto, la función de transferncia de la planta se determina por la ecuación 1, considerando los valores máximos de tensión y temperatura, se representan en la ecuación 4.

$\frac{c(s)}{u(s)}=\frac{4.0891 e^{-47.426 s}}{321.63 \mathrm{~s}+1}$

En la tabla 3, se muestran los rangos de entrada y salida de cada bloque.

\begin{tabular}{|l|r|r|}
\hline \multicolumn{1}{c}{ Bloque } & \multicolumn{1}{c|}{ Entrada } & \multicolumn{1}{c|}{ Salida } \\
\hline Referencia & & $0-600\left[{ }^{\circ} \mathrm{C}\right]$ \\
\hline Error & $0-30\left[\mathrm{~V}_{\mathrm{DC}}\right]$ & $0-20\left[\mathrm{~V}_{\mathrm{DC}}\right]$ \\
\hline Control On-Off & $0-20\left[\mathrm{~V}_{\mathrm{DC}}\right]$ & $0-5\left[\mathrm{~V}_{\mathrm{DC}}\right]$ \\
\hline Planta & $0-120\left[\mathrm{~V}_{\mathrm{AC}}\right]$ & $0-600\left[{ }^{\circ} \mathrm{C}\right]$ \\
\hline Sensor & $0-600\left[{ }^{\circ} \mathrm{C}\right]$ & $0-5\left[\mathrm{~V}_{\mathrm{DC}}\right]$ \\
\hline
\end{tabular}

Tabla 3 Rango de entradas y salidas Fuente: Elaboración Propia

Después, se diseño el sistema de control a lazo cerrado On-Off en relación a los rangos de entrada y salida. En la figura 3, se representan las etapas del sistema de control; el valor de referencia es una señal analógica que se introduce a una entrada analógica del microcontrolador ATMEGA 2560, en otras palabras, si el error es mayor a cero la señal de salida del controlador es $5 \mathrm{~V}_{\mathrm{DC}}$, en caso contrario, es decir, el valor del error es menor a cero la salida del controlador es cero, en relación a las ecuaciones 2 y 3 se obtienen:

$$
\begin{aligned}
& U(t)=U_{1}=5 V_{D C} \text { para } e(t)>0 \\
& U(t)=U_{2}=0 V_{D C} \text { para } e(t)<
\end{aligned}
$$

Cuando el error es mayor a cero se cumple la ecuación 5 , se obtiene una señal digital máxima de salida del control On-Off seguidamente se excita a los actuadores e inmediatamente se habilita a la planta que incrementa la temperatura hasta alcanza el valor de referencia y posteriormente se compara constantemente el valor de referencia y retroalimentación, cuando el error es menor a cero la salida del control On-Off conmuta de 5 $\mathrm{V}_{\mathrm{DC}}$ a $0 \mathrm{~V}_{\mathrm{DC}}$, por lo tanto los actuadores se desenergizan, por consiguiente, la planta no trasmite energía calorífica al molde, consiguientemente se repite el ciclo, ver figura 3.

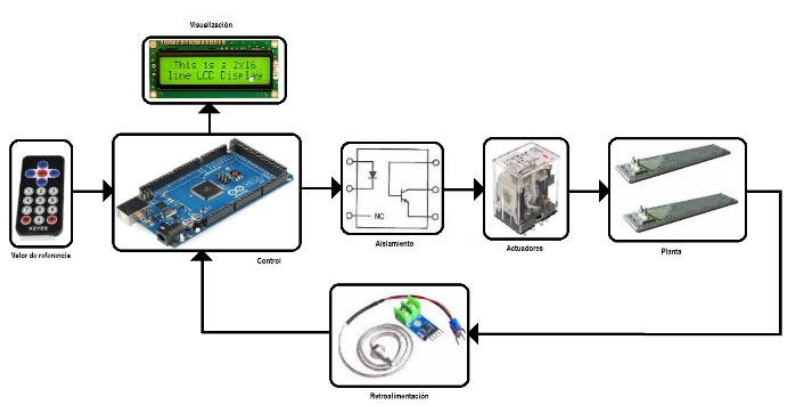

Figura 3 Sistema de control a lazo cerrado On-Off Fuente: Elaboración Propia

En la figura 4 y 5 , se muestras las curvas del comportamiento del sistema a lazo cerrado On-Off, donde el valor de referencia es $140{ }^{\circ} \mathrm{C}$ y $160{ }^{\circ} \mathrm{C}$. El periodo de tiempo activo es $48 \mathrm{seg}$ y $175 \mathrm{seg}$ en el tiempo bajo. Por lo tanto, el periodo de tiempo de oscilación de $223 \mathrm{seg}$

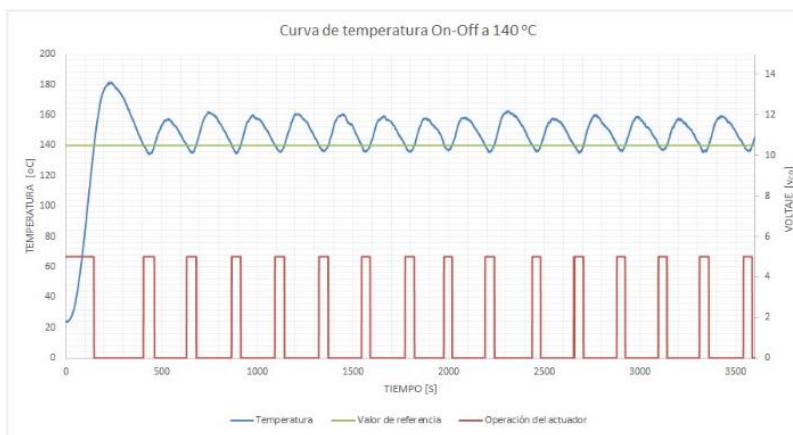

Figura 4 Curvas comportamiento del sistema de control On-Off con un valor de referencia $140^{\circ} \mathrm{C}$

Fuente: Elaboración Propia
ROSALES-DAVALOS, Jaime, ENRIQUEZ-PEREZ, Ma. de los Ángeles, LÓPEZ-RAMÍREZ, Roberto y MASTACHE-MASTACHE, Jorge Edmundo. Sistema de control de temperatura on-off implementado en el proceso de termoformado para elaboración de bloque a partir de envases multicapas. Revista de Ingeniería Eléctrica. 2019 


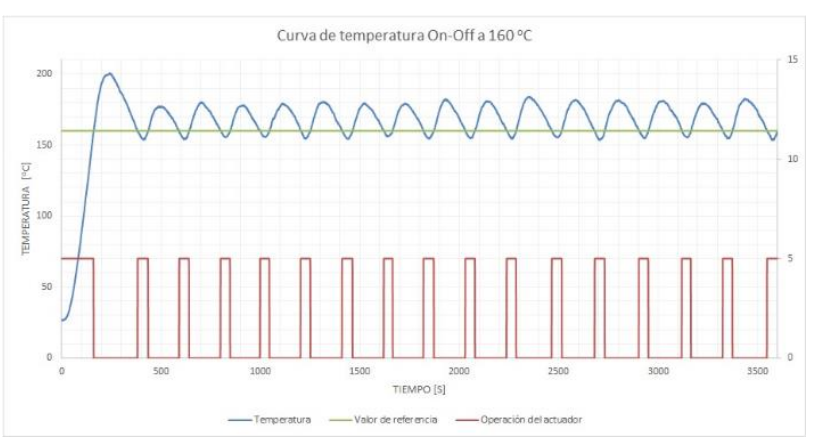

Figura 5 Curvas comportamiento del sistema de control On-Off con un valor de referencia $160^{\circ} \mathrm{C}$

Fuente: Elaboración Propia

\section{Agradecimientos}

Al Tecnológico de Estudios Superiores de Jocotitlán por el apoyo económico y sus instalaciones

\section{Conclusiones}

Se obtuvieron los bolques para la construcción de muro reciclando los envases multicapa, implementando el sistema de control On-Off en el proceso de termofomado, donde se controlo la temperatura de las resistencias eléctricas acopladas en cada uno de placas laterales, inferior y superior en el exterior molde, por consecuencia se manipula la temperatura en el interior del molde establecida entre $170{ }^{\circ} \mathrm{C}$ a 180 ${ }^{\circ} \mathrm{C}$ en un tiempo de 20 minutos.

Una ventaja del sistema de control, es poder manipular seis resistencias eléctricas de corriente alterna con el sistema de control; aunque no todas las resistencias eléctricas aumentan proporcinalmente la temperatura con respecto al tiempo, considerando las características eléctricas que las resitencias son semejantes.

\section{Referencias}

Benjamin, K. (1996). Sistemas de Control Automatico. México: Prentice Hall.

Bernard, J. (1999). Ciencias Ambientales y Desarrollo Sostenible. México: Pearson.

Inche Mitma, J., Vergiú Canto, J., Mavila Hinojoza, D., Godoy Martínez, M., \& Chung Pinzás, A. (2004). Diseño y evaluación de una planta de reciclaje de envases Tetra Pak a pequeña escala. Industrial Data, 7-17.
INEN, (2010). Norma 2542. Tolerancias en dimensiones. Laminas plasticas. Requsitos. Obtenido de http://www.normalización .gob.ec

Jiang, W., \& Jiang, X. (2012). Design of an Intelligent Temperature Control System Based on the Fuzzy Self-tuning PID. Procedia Engineering, 307-311.

Martínez , L. E., \& Moya, F. A. (2015). Diseño y construcción de una maquina termoformadora de plastico con control automatico para la empresa Miviltech Soluciones Industriales S. A.

Martínez Barrera, G., Hernández Zaragoza, J. B., López Lara, T., \& Menchaca Campos, C. (2015). Reciclaje de Plásticos, Materiales sustentables y reciclados en la construcción. OmniaScience, 124.

Ogata, K. (1998). Ingeniería de Control Moderna. Prentice Hall.

PLASTIGLAS. (1990). Manual Técnico TERMOFORMADO.

Tetra Pak busca aumentar su tasa de reciclaje. (01 de 07 de 2019). El Economista. Obtenido de http;//www.eleconomista.com.mx/empresas/Tet ra-Pak-busca-aumentar-su-tasa-de-reciclaje20190107-0100.html

Wang, S. (2012). Resistance Furnace Temperature System on Fuzzy PID Controller. . Journal of Information \& Computational Science, , 2627-2634. 